\title{
Chemical characterisation of the fracture surfaces of polyester resin and a polyester based nanocomposite
}

\author{
Surrey Materials Institute and Faculty of Engineering \& Physical Sciences, \\ University of Surrey, Guildford, Surrey, GU2 7XH, UK \\ Running head: \\ Aspects of a nanocomposite interface \\ Corresponding author: \\ Arran Wood \\ Address: \\ University of Surrey \\ FEPS A1 \\ Guildford \\ Surrey \\ GU2 7XH \\ $\mathrm{UK}$ \\ Telephone: $\quad$ + $\quad$ + $\quad$ + $\quad$ (0)1483 689627 \\ Fax: $\quad+44(0) 1483686291$ \\ E-mail address: $\quad$ a.wood@surrey.ac.uk
}

Arran R. Wood, Marie-Laure Abel, Paul A. Smith, John F. Watts 


\begin{abstract}
A novel in situ fracture stage has been used to conduct micro compact tension (microCT) tests within the preparation chamber of a time-of-flight secondary-ion mass spectrometry (ToF-SIMS) instrument. Tests have been undertaken in the usual ultra high vacuum (UHV) conditions and in an ambient air atmosphere for comparison. A plain polyester resin system has been compared to an organically modified silica (ormosil) nanoparticulate reinforced polyester resin nanocomposite. The force/displacement data are recorded permitting critical stress intensity factor, $\mathrm{K}_{\mathrm{IC}}$, determination. Comparative surface analysis of the samples fractured in air and in UHV revealed that fracture in UHV prevented atmospheric contamination and adsorption of adventitious hydrocarbon material, particularly airborne silicone oils. This led to superior spectral quality and improved interfacial chemistry interpretation, and has allowed the development of a model of failure in which the integrity of the interface between nanoparticulate and polyester resin remains intact when failure occurs. Such an observation is extremely important given the current level of interest in the mechanical behaviour of systems of this type. Adopting such a methodology has enabled nanomodified polymer systems to be investigated extending the potential application of ToF-SIMS as a useful fractographic tool in the field of nanocomposite analysis.
\end{abstract}

KEYWORDS: Nanocomposite; ToF-SIMS; In situ fracture; Ormosils 


\section{INTRODUCTION}

Advanced fibre reinforced composites (FRCs) often use highly cross linked epoxy and polyester resins as matrix materials. A limiting factor in their use is the relative brittleness of the matrix materials and the tendency for poor resistance to crack initiation and propagation [1]. Over the past 30 years there has been a great deal of interest in improving the mechanical properties, notably the fracture toughness, of polymeric matrices by the addition of various micrometer-sized modifying phases to the resin system. Various modifying phases have been investigated, phenolic beads [2], carbon beads [3] and rubber particles [4,5] for example.

More recently, with the development of nanotechnology, much attention has been given to replacing micrometre-sized with nanometre-sized modifying phases. Nanomaterials potentially offer higher improvements because they have significantly higher specific surface areas compared to their micro-phase counterparts, this, in turn can lead to the requirement for lower modifying phase loadings. This in turn can lead to reduced disruption of the primary reinforcement. It must also be considered that novel fracture mechanisms may exist on the nanoscale as we attempt to characterise nanomodifed material behaviour.

The interface is a critical region in any composite material. The success or failure of the composite will depend upon the nature of the interface as load is transferred across one phase to another, whether that is the interface of an adhesive joint, the bond strength of a fibre to the matrix in FRCs, or the effect of precipitates in metal alloys. Understanding the behaviour of such interfaces and their influence on mechanical properties is very important.

Surface analyis techniques have been used previously to investigate interfaces and as a tool in fractographic studies to help inform about the fracture mechanism and determine the locus of failure in FRCs [6-8]. XPS and ToF-SIMS provide the most suitable tools to investigate polymer surface chemistry because of their high chemical specificity and surface sensitivity. ToF-SIMS has the added advantage of providing information regarding molecular species, and hence good diagnostics for composite material component identification while using a smaller spot size than XPS at the current best performance under ideal conditions XPS is approximately $10 \mu \mathrm{m}$ compared with $100 \mathrm{~nm}$ for a high spatial resolution ToF-SIMS analysis.

It is well known that when utilising nanoscaled reinforcements, surface effects become critically important to the overall performance of the composite, therefore understanding the surface interaction in the composite or the interfacial chemistry between constituents is highly desirable.

The benefits of utilising surface analysis techniques to investigate nanoreinforced composites are therefore apparent. However, identifying signal from nanoreinforcements within a composite material using conventional surface analysis techniques is very challenging. Even with the most state-of-the-art surface analysis equipment the realistic 
analysis spot size that can be achieved on less than ideal samples is around $250 \mathrm{~nm}$ for ToF-SIMS and typically $50 \mu \mathrm{m}$ for XPS and an analysis depth typically between 1-5 $\mathrm{nm}$. With the size of reinforcing phases reducing into the nanoscale, $100 \mathrm{~nm}$ or less, and the capability to use lower loading levels, typically below 10 vol. \%, (lower than that for micrometre-sized modifying phase counterparts), the secondary-ion yield from such small particles will be very low. It is therefore necessary to enhance the technique to give us the best opportunity to detect and analyse the interfacial chemistry of such nanocomposite materials.

As with all spectroscopic techniques, in both XPS and ToF-SIMS, there is a trade-off between spectral resolution and sensitivity. Thus if one requires the ultimate spectral resolution this can generally only be acquired at a sacrifice of sensitivity which in the case of microbeam techniques is related to the effective spatial resolution attainable. In the work described in this paper it is essential that ToF-SIMS spectra are acquired at the highest spectral resolution and the instrumental parameters used reflect this need. This means, in turn, that there is a compromise in spatial resolution. On the face of it this may seem to be less than ideal but it is also important to ensure that the analysis reflects a representative sample of, in this case, the material fracture surface. Thus by acquiring spectra from an area which is large, compared to the reinforcement size, it is possible to be confident that any analytical conclusions that are reached are relevant to a relatively large number of reinforcement particles.

A common problem when performing surface analysis of polymeric fracture surfaces is deposition of adventitious hydrocarbons and molecules from test machine lubricants present in the atmosphere of failure contaminating the fracture surface [9]. This is because upon fracture of polymeric materials, polymer chain scission occurs, creating highly reactive radicals on the fracture surface. These radicals react rapidly with surrounding molecules, the driving force of which is to reduce the surface free energy of the material, thus contaminating the fracture surface and compromising the quality of spectra obtained as discussed elsewhere [9]. Further contamination can occur from fracture debris, together with manipulation, handling and preparation of samples prior to analysis.

It is therefore beneficial if fracture can occur in a clean environment preferably within ultra high vacuum (UHV) preventing exposure of the fracture surface to atmospheric contaminants and thereby maintaining a pristine fracture surface for analysis. To this end we have developed an automated in situ fracture stage for use on our ToF-SIMS system to perform mechanical testing under UHV conditions. An automated linear drive controls the strain rate and a load cell interfaced with a data logger used to measure the load enabling force/displacement information to be recorded as the specimen is fractured. Upon failure the sample can be manipulated to the analysis chamber using wobble sticks where spectra of the fresh fracture surface can be obtained without exposure to atmosphere and risk of contamination [10].

In this paper we describe the use of the fracture stage to perform in situ micro-compact tension (micro-CT) tests on two types of material, a baseline polyester resin and an 
organically modified silica (ormosil) nanoparticulate reinforced polyester resin. CT testing is a standard mechanical test methodology to measure fracture toughness properties of materials. Samples of each material have been fractured in atmosphere and in UHV using the in situ fracture stage. Critical stress intensity factor values may be determined by analysis of the force/displacement data. ToF-SIMS spectra of the fracture surfaces are presented. Comparisons of the spectra reveal the in situ fractured samples produce contaminant free fracture surfaces which has enabled the nanoparticulate modifying phase to be located. This has enabled the use of ToF-SIMS as a tool to investigate the interfacial properties and fracture behaviour of nanocomposite materials to be explored.

\section{EXPERIMENTAL Materials}

Two types of material have been tested, the first, a baseline resin system, the second the baseline material modified with the addition of ormosil nanoparticles. The base resin was an unsaturated polyester resin, supplied without the addition of the silica thixotropic agent, (Crystic 2-406 PA, Scott Bader). Phenyl ormosil nanoparticles, produced via a modified Stöber hydrolytic route [11] were supplied and incorporated into the resin system to manufacture a 1 vol. \% nanomodifed material using a method described previously [12]. The resins were cast into moulds to produce $3 \mathrm{~mm}$ thick plaques. These resin plaques were cured for 24 hours at room temperature then received a 3 hour postcure at $80^{\circ} \mathrm{C}$.

\section{Mechanical Testing}

Micro-CT specimens were fashioned from the resin plaques in accordance with ASTM $\mathrm{D} 5045$, with $\mathrm{W}=12 \mathrm{~mm}$. The specimens were pre-cracked by tapping a fresh razor blade into a saw cut notch. The automated in situ fracture stage was used to conduct micro-CT testing using a displacement rate equivalent to $2.5 \times 10^{-6} \mathrm{~m} \mathrm{~s}^{-1}$. Details of the fracture stage are described elsewhere [10]. By way of comparison samples of both materials have been tested using the fracture stage in air and in UHV. Upon failure of the specimen the lower half of the specimen was removed from the load string, mounted in the sample platen and transferred to the analysis chamber for ToF-SIMS analysis. For the samples tested in UHV this could be done by manipulating the samples using wobble sticks therefore preventing exposure to atmosphere.

\section{Scanning Electron Microscopy}

Scanning electron microscopy (SEM) was carried out using a Hitachi S-3200 microscope. Prior to SEM analysis (which was always undertaken after ToF-SIMS analysis) specimens were sputter coated with fine grained gold to a thickness of $5 \mathrm{~nm}$ to prevent electrostatic charging. An accelerating potential of $20 \mathrm{kV}$ was used for all SEM studies described in this paper. 


\section{Surface Analysis}

ToF-SIMS analysis was performed using a TOF.SIMS 5 (ION-TOF GmbH, Münster, Germany), fitted with a bismuth liquid metal ion source. Polyatomic $\mathrm{Bi}_{3}{ }^{+}$was used as the primary ion source using an accelerating voltage of $25 \mathrm{keV}$ and a primary ion current of $0.3 \mathrm{pA}$. The bunched mode was selected to obtain spectra with high mass resolution and a spot size of about $4 \mu \mathrm{m}$. Spectra were recorded for 60 seconds over 200 x $200 \mu \mathrm{m}$ area using a resolution of $128 \times 128$ pixels ensuring that the ion dose remained well below the notional static limit of $10^{12}$ ions $/ \mathrm{cm}^{2} /$ analysis.

\section{RESULTS AND DISCUSSION Mechanical data}

The force/displacement trace for the nanomodified resin micro-CT specimen tested within the sample preparation chamber at ambient pressure is shown in Figure 1. The critical stress intensity factor, $\mathrm{K}_{\mathrm{IC}}$, can be determined from the peak load and specimen geometry, as described in ASTM D5045. Data from a single sample has been included to demonstrate the methodology and advantage of being able to perform mechanical testing in situ. The main focus of this investigation, however, is not to characterise mechanical properties, which would require replicate specimens to establish adequate confidence limits, but rather to produce contaminant free fracture surfaces for surface analysis.

The following samples were also tested using the fracture stage, a plain resin sample fractured in air (PRA), plain resin fractured in UHV (PRV), nanoresin fractured in air (NRA) and nanoresin fractured in UHV (NRV) to produce contaminant free fracture surfaces for surface analytical comparison. Table 1 summarises these sample details.

\section{Surface Analysis - Overview of results for all samples}

The nanocomposite utilises phenyl ormosil nanoparticles to reinforce the base polyester resin system. One of the aims of the ToF-SIMS analysis is to determine whether there is any evidence of these nanoparticles in the mass spectrum of the nanocomposite fracture surface. Such information could lead to a more informed understanding of the interface behaviour between the resin and modifying phase and also the failure mechanism(s).

A previous study of the surface chemistry of the phenyl ormosil particles identified diagnostic peaks due to fragment ions from the phenyl ring at $39 \mathrm{u}^{*}\left(\mathrm{C}_{3} \mathrm{H}_{3}\right)$ and $55 \mathrm{u}$ $\left(\mathrm{C}_{4} \mathrm{H}_{7}\right)$, silicon containing peaks from the seed silica particle $28 \mathrm{u}(\mathrm{Si})$ and $45 \mathrm{u}(\mathrm{SiOH})$ and an increase in relative intensity of the sodium peak at $23 \mathrm{u}(\mathrm{Na})$ due to a feature of the ormosil production [13]. The polyester resin contains aromatic carbon, therefore peaks at $39 \mathrm{u}$ and $55 \mathrm{u}$ associated with the phenyl organic modification of the nanoparticles cannot be used as a unique diagnostic for the ormosil particles. Therefore the marker fragment ions indicating the presence of ormosils will be the silicon containing fragment ions. Any silicon containing contaminants, notably

\footnotetext{
${ }^{*} \mathrm{u}$ is unified mass unit, the IUPAC approved unit for mass spectrometry, defined as one twelfth of the mass of a carbon -12 atom in its ground state
} 
poly(dimethylsiloxane) (PDMS) will compromise the spectra and our ability to identify the ormosil nanoparticles.

ToF-SIMS analysis was performed on the fracture surfaces of the micro-CT specimens. Fracture surfaces can often have rough topography which makes surface analysis challenging. However, the relative brittle nature of polyester gave rise to relatively smooth flat fracture surfaces and a flat region was selected to perform the analyses.

The positive ToF-SIMS spectra for the four samples, polyester in ambient (PRA), and UHV (PRV), and nanomodified polyester in ambient (NRA), and UHV (NRV) between mass range 1-200 u can be seen in Figure 2(a-d) respectively. The spectra look qualitatively similar, as would be expected from initial observation, however characteristic peaks associated with PDMS fragment ions, $73 \mathrm{u}\left(\mathrm{SiC}_{3} \mathrm{H}_{9}\right)$ and $147 \mathrm{u}$ $\left(\mathrm{Si}_{2} \mathrm{C}_{5} \mathrm{H}_{15} \mathrm{O}\right)$ have been identified. It is clear that there are large PDMS peaks in the spectra of the samples fractured in air Figures 2(a) and 2(c).

A more detailed inspection of these spectra is necessary to determine their differences. Figure 3 is a high resolution view of spectra at a nominal mass $28 \mathrm{u}$. As identified in this figure, silicon is present in the spectrum of the PRA sample. This, together with peaks at $73 \mathrm{u}$ and $147 \mathrm{u}$ diagnostic of PDMS, allows us to attribute the silicon to PDMS contamination. The spectrum of the PRV sample, spectrum Figure 3(b), does not contain silicon. The spectra of the nanomodifed resins also identify silicon but the nanoresin sample fractured in air, Figures 2(c) and 3(c) also contains PDMS contamination and so the silicon cannot be attributed solely to the ormosil nanoparticles. The silicon in spectrum Figure 3(d) can however be used as diagnostic of the ormosil particles. These spectra all contain a $\mathrm{C}_{2} \mathrm{H}_{4}$ peak from the resin material. Interestingly the $\mathrm{CO}$ peak seems more intense for both samples fractured in UHV. It is proposed that this is because adventitious hydrocarbons have not been permitted to adsorb onto the surface, which would increase the hydrocarbon peak intensities, which will then dominate the other composite specific fragment ions within the spectrum.

Inspection of the spectra at nominal mass $73 \mathrm{u}$, Figure 4, clearly shows differences between the samples. The diagnostic PDMS fragment $\mathrm{SiC}_{3} \mathrm{H}_{9}$ dominates in the samples fractured in air, Figures 4(a) and 4(c). The samples fractured in UHV, Figures 4(b) and 4(d) have two peaks, $\mathrm{C}_{6} \mathrm{H}$, which is not observed in the other two spectra, and a dominant peak assigned to $\mathrm{C}_{3} \mathrm{H}_{5} \mathrm{O}_{2}$ from the polyester resin, at lower intensities than the PDMS peak observed in the samples fractured in air. The polyester peak is also present in Figures 4(a) and 4(c) however the PDMS fragment dominates at significantly higher intensity for sample PRA.

Similarly inspection of nominal mass $147 \mathrm{u}$, Figure 5, reveals that a peak of fragment ion $\mathrm{C}_{9} \mathrm{H}_{7} \mathrm{O}_{2}$ from the polyester is present. However this peak is dominated by the characteristic PDMS peak $\mathrm{Si}_{2} \mathrm{C}_{5} \mathrm{H}_{15} \mathrm{O}$ for the two samples fractured in air, Figures 5(a) and 5(c). 
This data clearly demonstrates the necessity to fracture the samples in situ in order to remove the prospect of PDMS contamination and therefore enable identification of the silicon containing fragment ions which are diagnostic of the ormosil nanoparticles. Comparison will therefore be made of the two samples fractured in vacuum, PRV and NRV, to identify their differences and investigate further the presence of the ormosil nanoparticles.

\section{Comparison of the in situ fracture surfaces}

Figure 6 shows the spectra for the two samples fractured in situ, (a) PRV and (b) NRV with labels identifying the major peaks and those characteristic of the polyester resin. Chemical structures for these peak assignments are presented in Table 2 [14-16].

Nominal mass 28 u has already been compared in Figure 3, which shows the presence of silicon, which indicates the presence of ormosils in the NRV sample but not the PRV sample. Comparison of the spectra at nominal mass $29 \mathrm{u}$, Figure 7 also shows that there is a small peak attributed to $\mathrm{SiH}$ from the ormosil nanoparticles in the nanoresin fracture surface which is not present in the plain resin sample.

Figure 8 compares the spectra at nominal mass $45 \mathrm{u}$ which also reveals $\mathrm{SiOH}$ diagnostic of the ormosil particles in the nanoresin sample but not the plain resin sample.

Spectra were also taken in the negative mode but are not presented here. These spectra provided similar information regarding the presence of silicon containing fragment ions in PRA, NRA and NRV. The origin of the silicon containing fragments in the two samples fractured in air cannot be solely attributed to the ormosil particles, as already discussed.

\section{Fracture Mechanisms}

Scanning electron micrographs ahead of the pre-crack of the micro-CT fracture surfaces for both the plain resin and the nanoresin samples fractured in vacuum, PRV and NRV, at various magnifications are presented in Figure 9. The two samples appear very similar. Both have relatively flat and smooth fracture surfaces with river line patterns characteristic of brittle polymer failures [17].

Having studied large areas of the fracture surfaces, it is apparent that there is a higher density of fracture features and river lines on the nanomodified system compared to the plain resin which is predominately smooth with a mirror finish. The increase in these fracture features in the nanoresin indicates a higher amount of fracture activity as the crack front propagates through the sample. This increase in activity can be associated with a small increase in plastic behaviour leading to an increase in fracture toughness, as discussed by Jesson et al [18] in a companion paper using identical phenyl modified ormosil nanoparticulate reinforced polyester resin. One possible reason for the increase in the fracture events in the nanoresin material is described by Engel et al [19]. They describe a mechanism of failure in brittle polymers whereby the crack front divides into 
several sections which reunite as the fracture progresses. Additionally, as the crack front passes over inhomogeneities, (such as nanoparticles or agglomerations of nanoparticles present in the nanoresin sample used in this investigation), the cleavage stops and chips can splinter off, similar to the features observed in Figure 9(d). We note in passing that the micrographs in that paper appear different to those presented here. It is suggested that these differences are associated with the use of a different test specimen (DEN in Jesson et al, CT here) which may promote some differences in mechanical behaviour.

The ToF-SIMS data reported thus far demonstrates the benefits of conducting failure within UHV, however it does not directly provide information regarding the fracture mechanism(s) in the nanocomposite. In order to do this additional ToF-SIMS analysis, using the same parameters described above, has permitted high mass resolution spectra to be obtained for a sample of the phenyl modified ormosil nanoparticles to complement previous reference data, as presented in Figure 10(b) and compared to the NRV fracture surface 10(a). The dominant peaks from the spectrum and possible structures are presented in Table 3.

As indicated above the polycyclic aromatic fragment ions from the phenol rings and recombination of the phenyl groups forming hydrocarbon peaks cannot be used as markers for the ormosil because they are also present in the polyester resin, so it is the silicon containing fragments which are of interest. A more detailed comparison of the NRV sample and the characteristic ormosil peaks are presented in Figure 11.

Figure 11(a) and 11(b) compare the spectra of the nanomodifed resin fracture surface and the phenyl ormosil nanoparticles at nominal mass $28 \mathrm{u}$. The presence of silicon is clearly observed in both spectra. The silicon peak is significantly higher in the spectrum of the ormosil nanoparticles as would be expected as there is a far higher volume of silicon containing material present. There is also a peak associated with the fragment ion $\mathrm{C}_{2} \mathrm{H}_{4}$ which is attributed to fragmentation of hydrocarbon material, from the polyester resin in Figure 11(a), and at a much lower intensity peak in Figure 11(b) for the ormosils, where the fragment is probably caused by fragmentation of the phenyl ring.

Figure 11(c) and 11(d) compare the spectra at nominal mass $29 \mathrm{u}$. The spectrum of the nanomodifed resin, Figure 11(c), has peaks associated with fragmentation of hydrocarbon material. Figure 11(d) has peaks of the isotope of silicon 29, and $\mathrm{SiH}$, which are undetected in the nanoresin spectrum. There is a relatively lower intensity peak for CHO. This is probably because there is less oxygen containing material in the ormosil sample compared to that of the nanoresin sample.

The spectrum for the two samples is compared at nominal mass $45 \mathrm{u}$ in Figure 11(e), the nanoresin, and 11(f), the ormosils. The nanoresin spectrum, Figure 11(e), has a very small peak of $\mathrm{SiOH}$ and $\mathrm{CHO}_{2}$, but is mainly dominated by the $\mathrm{C}_{2} \mathrm{H}_{5} \mathrm{O}$ from the polyester material. Only $\mathrm{SiOH}$ is present in the spectrum of Figure 11(f).

Comparison at nominal mass $53 \mathrm{u}$ has peaks associated with fragmentation of the polyester material within the nanoresin, Figure $11(\mathrm{~g})$. A large $\mathrm{SiC}_{2} \mathrm{H}$ peak and $\mathrm{a}_{4} \mathrm{H}_{5}$ 
peak from the fragmentation of the phenyl ring are present in the ormosil spectrum, Figure 11(h).

Figure 11(i) has peaks associated with the fragmentation and recombination of the polyester, $\mathrm{C}_{7} \mathrm{H}_{5} \mathrm{O}$ and $\mathrm{C}_{8} \mathrm{H}_{9}$. Figure $11(\mathrm{j})$ has one large peak of $\mathrm{SiC}_{6} \mathrm{H}_{5}$, a phenyl ring attached to a silicon atom, the main modification of the ormosil nanoparticles.

Figure $11(\mathrm{k})$ has one polycyclic aromatic peak, $\mathrm{C}_{10} \mathrm{H}_{9}$, attributed to the fragmentation and recombination of the polyester. Figure 11(1) has a silicon containing peak formed from the fragmentation and recombination of the ormosil material.

There are several significant observations that can be made from these spectra. Firstly the only silicon containing peaks, characteristic of the ormosil nanoparticles observed in the nanoresin fracture surface are the silicon peak itself at $28 \mathrm{u}$, Figure 11(a), and also a very small $\mathrm{SiOH}$ peak, as seen in Figure 11(e). None of the other silicon containing peaks, characteristic of the ormosils, are observed in the spectrum of nanoresin fracture surface.

There are several reasons for this. The most significant is the comparison at nominal mass $105 \mathrm{u}$. Figure 11(j) shows the large $\mathrm{SiC}_{6} \mathrm{H}_{5}$ peak at $105 \mathrm{u}$ which is not present in the nanocomposite fracture surface, Figure 11(i). This is unsurprising given the functionality is very similar to that of the polyester matrix material. This suggests that there is an interaction with the polyester resin and that the ormosil particles bond with the polyester resin. Similarly there is only a very small peak at $45 \mathrm{u}$ associated with $\mathrm{SiOH}$ in the nanocomposite fracture surface, Figure 11(e), compared to that of the ormosils, Figure 11(f). This again suggests that the silanol functionality interacts with the resin system. These peaks are associated with the silica nanoparticle, not the organic modification on the surface of the ormosils, which bonds with the polyester due to their functional similarity.

A reason for these observations is that the analysis depth for atomic ions is higher than that of larger molecular fragments. The inability to detect the large characteristic fragments of the ormosil yet still be able to detect signal from the silicon and the $\mathrm{SiOH}$ in the spectrum of the fracture surface of the nanoresin, suggests that the nanoparticles lie buried just beneath the fracture surface.

These observations suggest that the fracture path runs around the nanoparticles, leaving a thin overlayer of interacting interphase between the polyester and phenyl functionality on the ormosil surface within the nanomodifed resin fracture surface. Peaks associated with the interacting fragments are not detected in the ToF-SIMS spectra, neither are the high molecular weight fragments which cannot be detected through the resin overlayer coverage.

These interactions have previously been studied using inverse gas chromatography (IGC) and X-ray photoelectron spectroscopy (XPS) [20]. A schematic of these interactions is shown in Figure 12. Here it was suggested that the silanol groups form hydrogen bonds with the polyester resin and the phenyl moiety on the silica nanoparticle could form $\pi \rightarrow$ 
$\pi^{*}$ interactions with the phenyl rings of the polyester resin, similar to those found in between graphene sheets.

On the basis of this ToF-SIMS data and previous studies on the interaction between phenyl modified ormosil nanoparticles and polyester resin it can be suggested that specific, but relatively weak, interactions bond the nanoparticles with the polyester resin.

Fracture through the nanocomposite does not reveal any of the functional groups observed on the nanoparticle surface. The spectrum for the nanocomposite is dominated by peaks characteristic of the polyester resin. The silicon elemental and very small silicon fragment ions observed in the fracture surface do come from the ormosil nanoparticles, however the depth of analyses for such small inorganic fragment ions is deeper than for molecular fragments. This therefore suggests that there is a resin overlayer around the ormosil nanoparticles which lie just beneath the fracture surface. The functional groups of the ormosil are not observed in the ToF-SIMS spectra of the nanocomposite fracture surface because these form interactions with the polyester resin to form an interpenetrating interphase region.

The fracture path therefore goes through and around the interphase region between nanoparticle and matrix which acts to absorb energy and hence toughen the composite material. This has been determined with more complete fracture mechanics investigation by Jesson et al [18].

\section{Conclusions}

The benefits of performing in situ fracture have been demonstrated using micro-CT samples of polyester and a phenyl ormosil modified polyester nanocomposite. Conducting testing in UHV prevented the adsorption of adventitious hydrocarbon material and contaminants from the atmosphere onto the highly reactive polymer fracture surface which compromise the spectra quality. Removing the potential for contamination permitted the origin of the silicon peak observed in the NRV sample to be wholly attributed to the nanomodifying ormosils.

Detailed inspection of the ToF-SIMS spectra then provided information regarding the interfacial behaviour and interaction between the phenyl modified ormosil nanoparticles and the polyester matrix material. Comparison of the spectra obtained of the nanocomposite fracture surface and the ormosil nanoparticles then permitted an indication of the fracture mechanism of the nanocomposite.

The future challenge for this type of analysis will be to develop imaging techniques in order to map the fracture surface to locate the nanoparticles. Mass resolved images of the diagnostic ormosil peaks potentially enable a chemical map of the fracture surface and could be used to locate individual nanoparticles on the fracture surface. However the low signal generated from these nanoparticles and the necessity for high mass resolution in order to identify them, means that an increased spot size must be used. These factors 
make ToF-SIMS imaging of nanocomposites difficult, but achievable with improved analysis methods and data analysis techniques.

\section{ACKNOWLEGEMENTS}

ARW wishes to thank the EPSRC and University of Surrey for funding and Dr Steve Hinder for assistance with ToF-SIMS analysis.

\section{REFERENCES}

1. M. Zhang and R. P. Singh, Mater. Lett. 58, 408-412 (2004)

2. J. J. Lee, S. L. Ogin and P. A. Smith, Particulate Reinforced Matrices for Continuous Fibre Composites, Proceedings, $5^{\text {th }}$ PRI International Conferrence on Fibre Reifnrced Composites, FRC ' 92, pp 22/1-22/10, University of Newcastle upon Tyne, ( 1992)

3. J. J. Lee, S. L. Ogin and P. A. Smith, in: Composite Materials: Fatigue and Fracture, R. H. Martin (Ed.) Vol. 5, pp. 38-60. American Society for Testing and Materials, Philadelphia (1995)

4. P. D. Tetlow, J. F. Mandell and F. J. McGarry, in: Proceedings of the $34^{\text {th }}$ Annual Technical Conference, Reinforced Plastics/Composites Institute, Section 23-F, pp. 1-4 (1979)

5. A. J. Kinloch, D. L. Maxwell and R. J. Young, J. Mater. Sci. 20, 4169-4184 (1985)

6. J. E. Castle and J. F. Watts, in: Interfaces in Polymers, Ceramics and Metal Matrix Composites, H. Ishida (Ed.), Proceedings of Composite Interfaces International Conference II, pp. 57-71, Cleveland (1988)

7. P. M. A. Sherwood, in: Handbook of Surface and Interface Analysis: Methods for Problem-Solving, J. C. Riviere and S. Myhra (Eds.), Marcel Dekkar Inc., New York (1998)

8. P. Denison, F. R. Jones, A. Brown, P. Humphrey and J. Harvey, J. Mater. Sci. 23, 2153-2156

9. A. C. Prickett, P. A. Smith and J. F. Watts, Surf. Interface Anal. 31, 11-17 (2001)

10. A. R. Wood, N. Benedetto, N. Hooker, E. Scullion, P. A. Smith and J. F. Watts, Surf. Interface Anal., submitted (2008)

11. A. Arkhireeva and J. N. Hay, J. Mater. Chem. 13, 3122-3127 (2003)

12. D. A. Jesson, The interaction of nano-composite particles with a polyester resin and the effect on mechanical properties, PhD Thesis, School of Engineering, University of Surrey, UK (2004)

13. D. A. Jesson M-L. Abel, A. Arkhireeva, J. N. Hay, P. A. Smith and J. F. Watts, Surf. Interface Anal. 36, 765-768 (2004)

14. The Static SIMS Library, Part 2 - Organic Materials, SurfaceSpectra Ltd., Manchester, UK, Wiley, 1999

15. The Münster High Mass Resolution Static SIMS Library, Edition V1.3, Vol. II Polymers

16. Static SIMS Handbook of Polymer Analysis, Perkin-Elmer Corporation 
17. D. Hull, Fractography: Observing, Measuring and Interpreting Fracture Surface Fractography, Cambridge University Press, Cambridge (1999), p 91

18. D. A. Jesson, P. A. Smith, J. N. Hay and J. F. Watts, J. Mater. Sci 42, 3230-3237 (2007)

19. L. Engel, H. Klingele, G. W. Ehrenstein and H. Schaper, An Atlas of Polymer Damage, Wolfe Publishing Ltd, London (1981), p 177

20. D. A. Jesson, M-L. Abel, J. N. Hay, P. A. Smith and J. F. Watts, Langmuir 22, 5144-5151 (2006) 
Figures and Tables

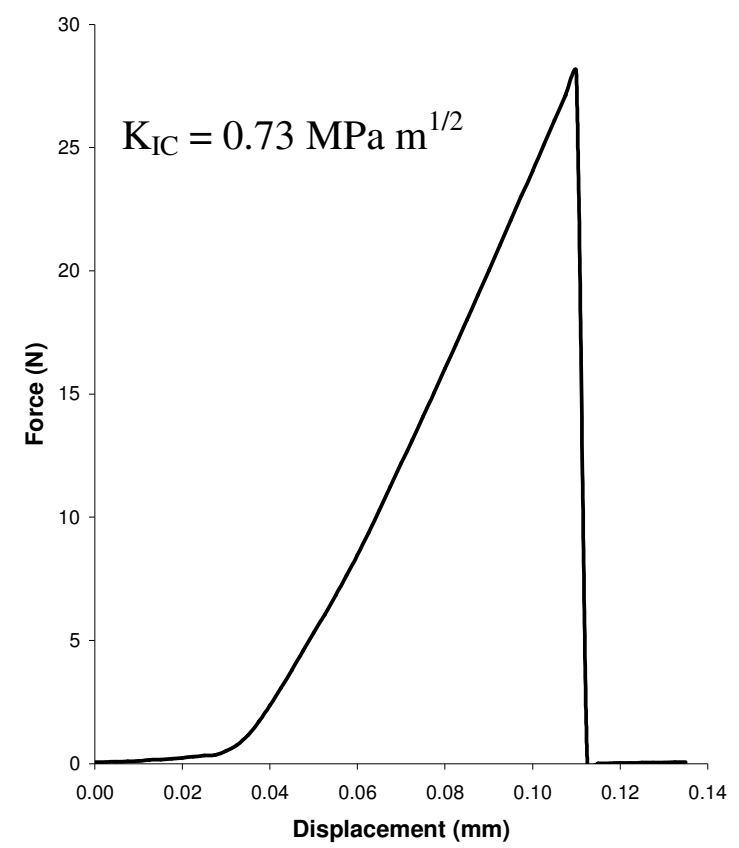

Figure 1. Micro-compact tension force/displacement plot for NRA sample

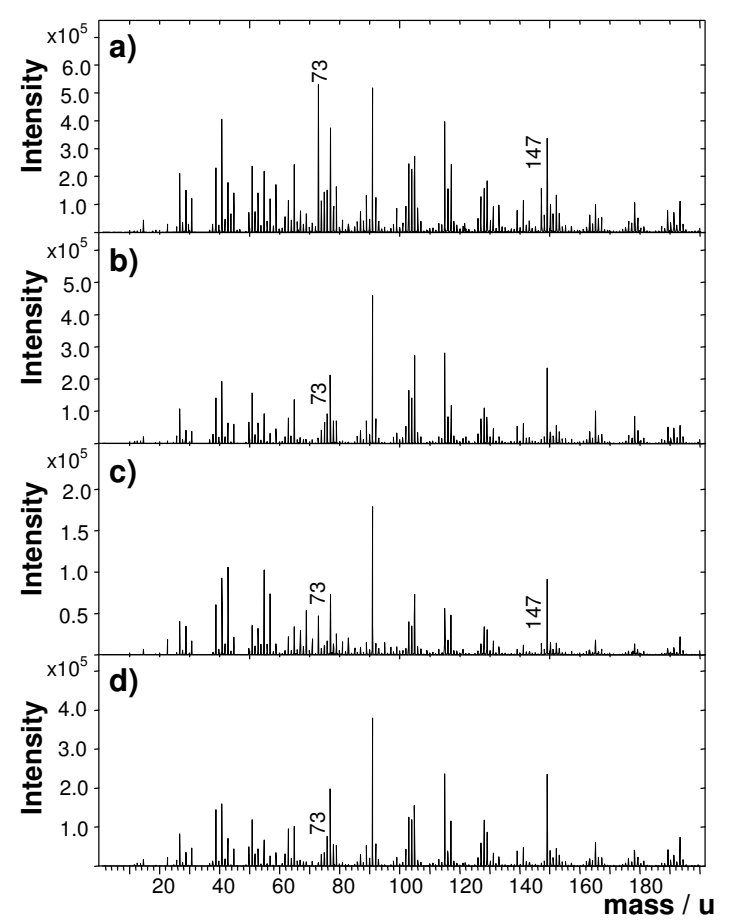

Figure 2. Positive spectrum of nominal mass range 1-200 $u$ for a) PRA, b) PRV, c) NRA, d) NRV. The nominal PDMS characteristic peaks are labelled, $73 \mathrm{u}$ and $147 \mathrm{u}$ 


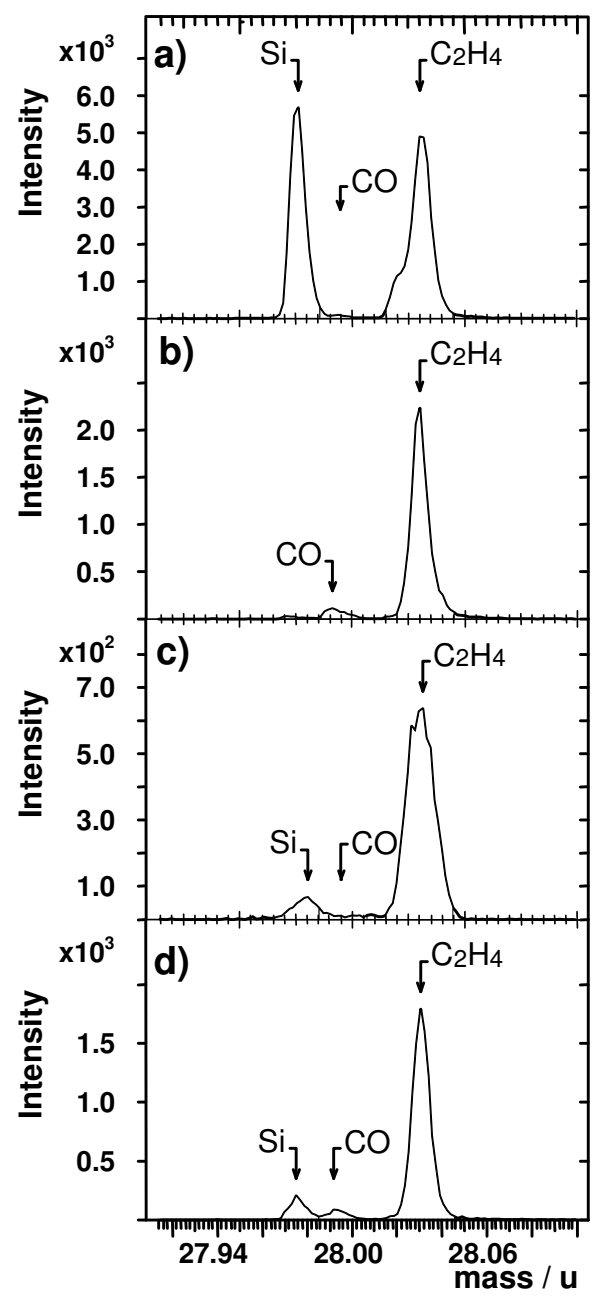

Figure 3. Positive spectrum of nominal mass $28 \mathrm{u}$ for a) PRA, b) PRV, c) NRA, d) NRV 


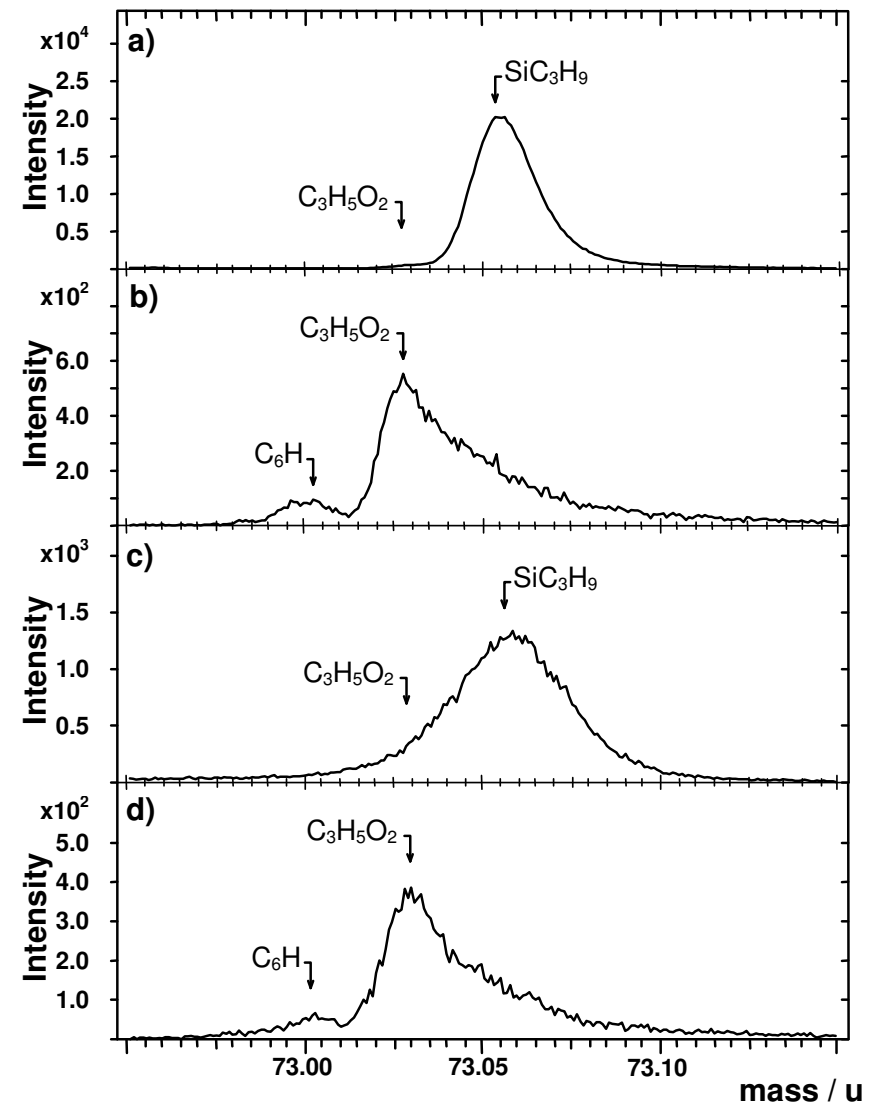

Figure 4. Positive spectrum of nominal mass 73 u for a) PRA, b) PRV, c) NRA, d) NRV 


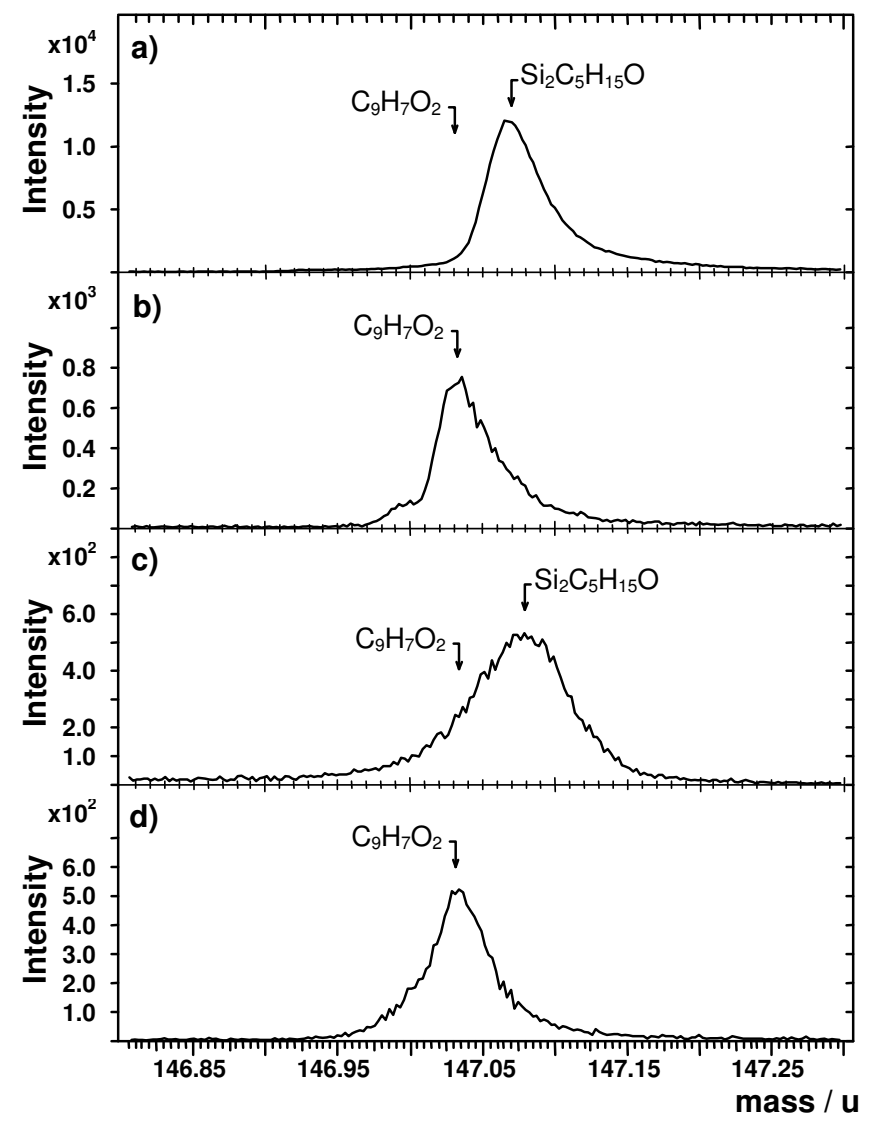

Figure 5. Positive spectrum of nominal mass $147 \mathrm{u}$ for a) PRA, b) PRV, c) NRA, d) NRV

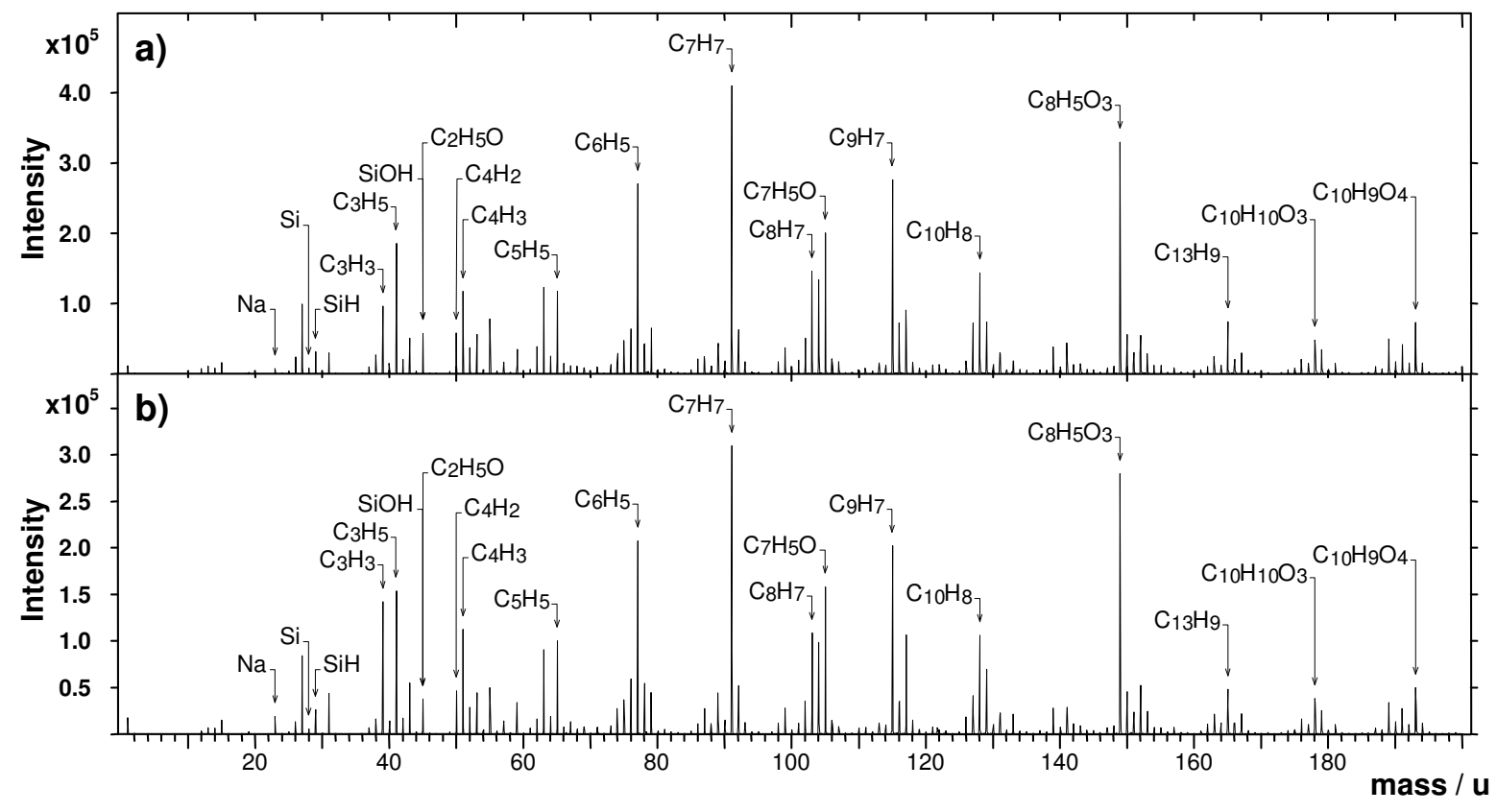

Figure 6. Positive spectrum of nominal mass range 1-200 u for a) PRV, b) NRV 


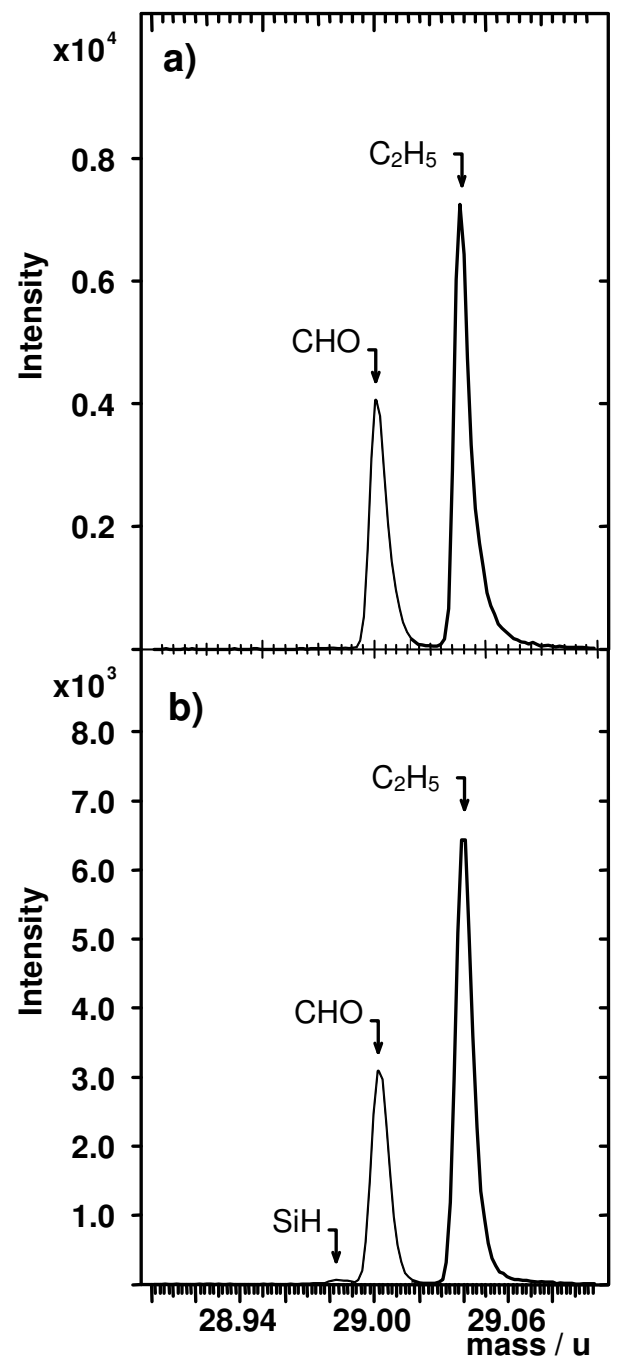

Figure 7. Positive spectrum of nominal mass $29 \mathrm{u}$ for a) PRV, b) NRV 


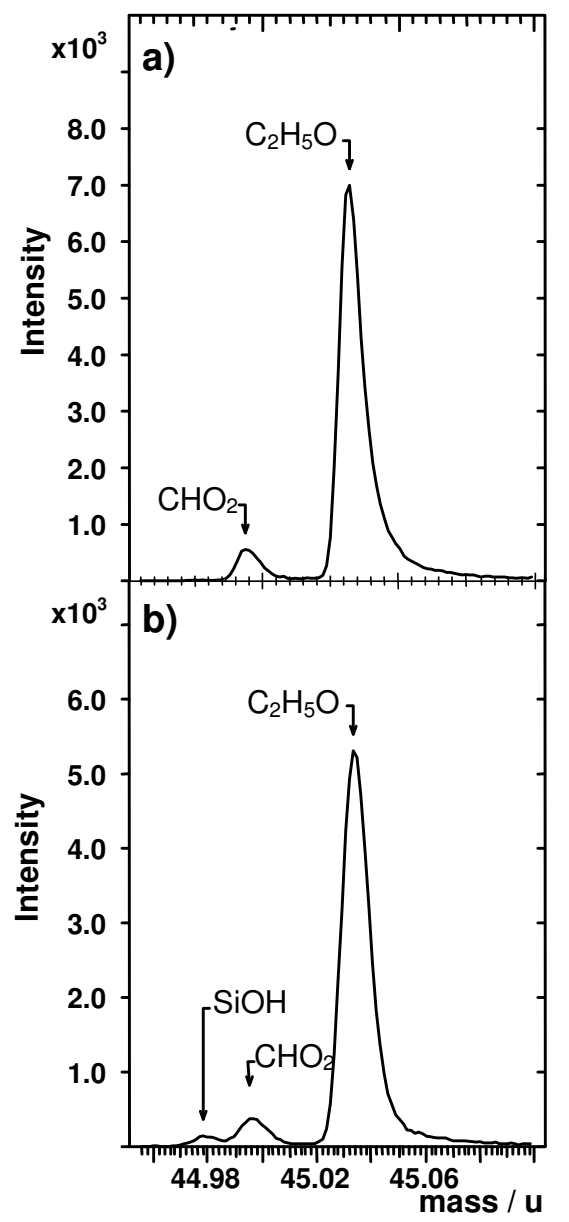

Figure 8. Positive spectrum of nominal mass $45 \mathrm{u}$ for a) PRV, b) NRV
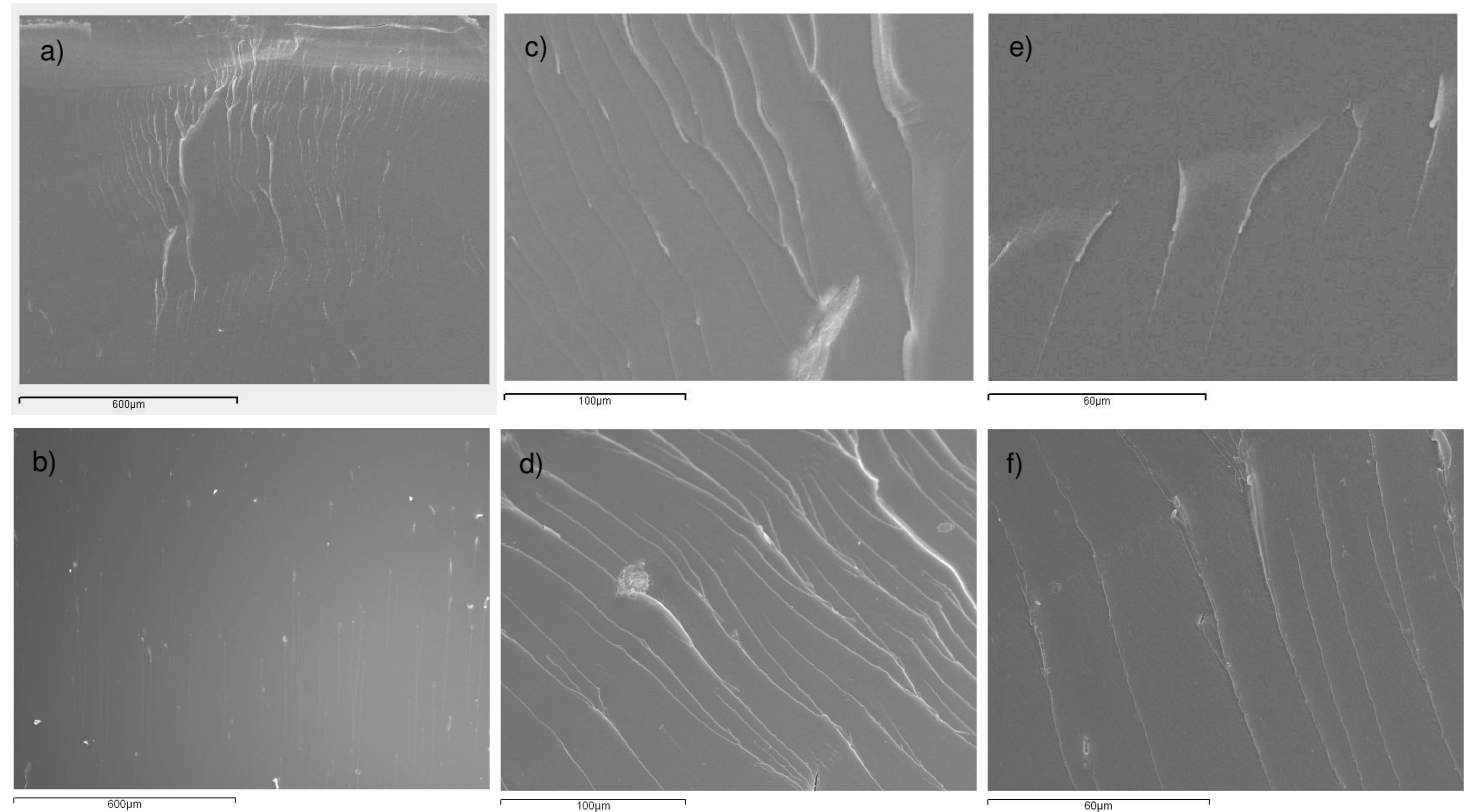

Figure 9. Scanning electron micrographs of PRV (a),(c),(d) and NRV (b),(d),(f) at $\mathrm{x} 100, \mathrm{x} 500$, and $\mathrm{x} 1000$ magnifications respectively. The crack direction runs from top to bottom in all the micrographs. 


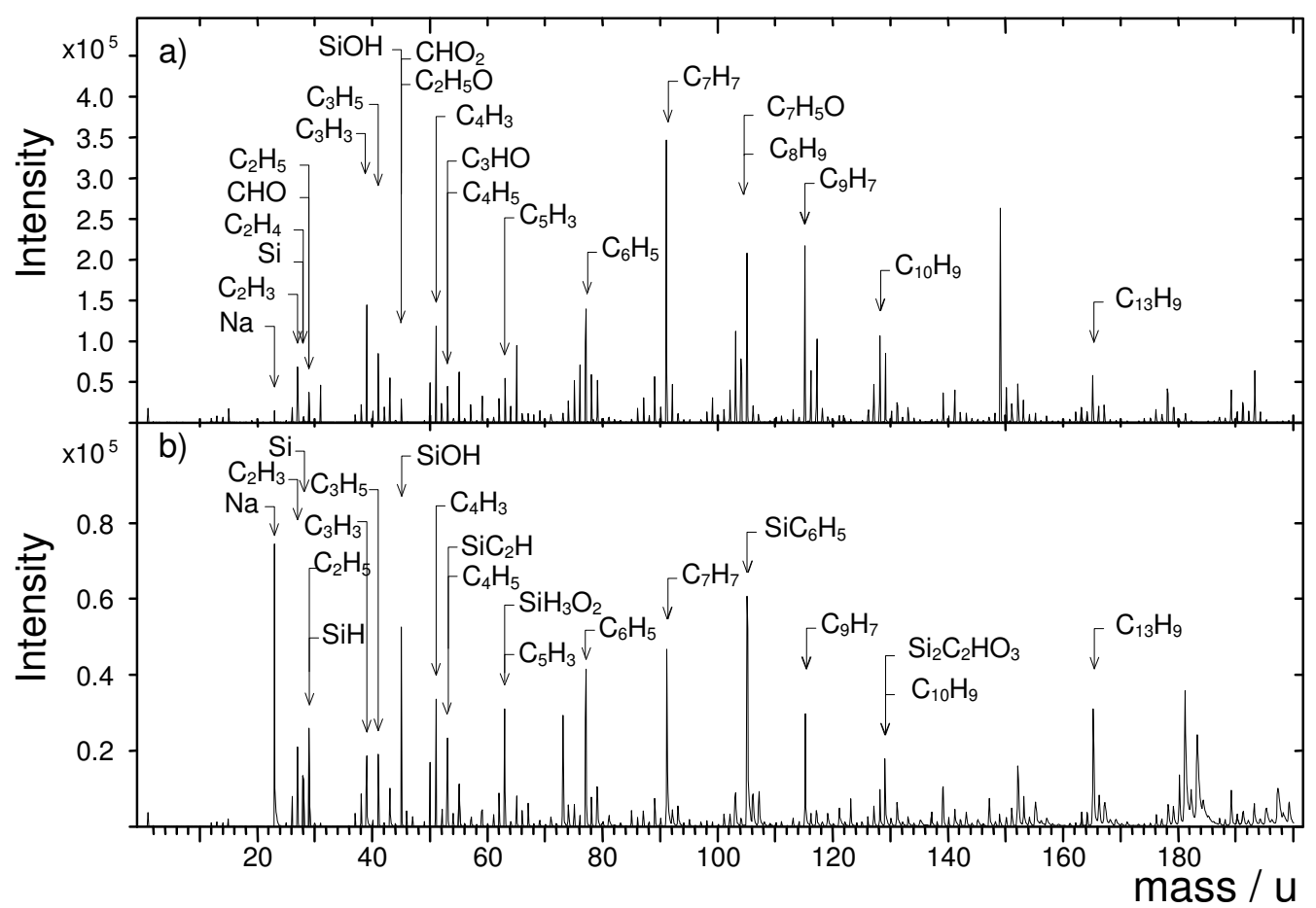

Figure 10. Comparison of the positive ToF-SIMS spectra between 1-200 u for a) NRV fracture surface and b) phenly modified ormosil nanoparticls 

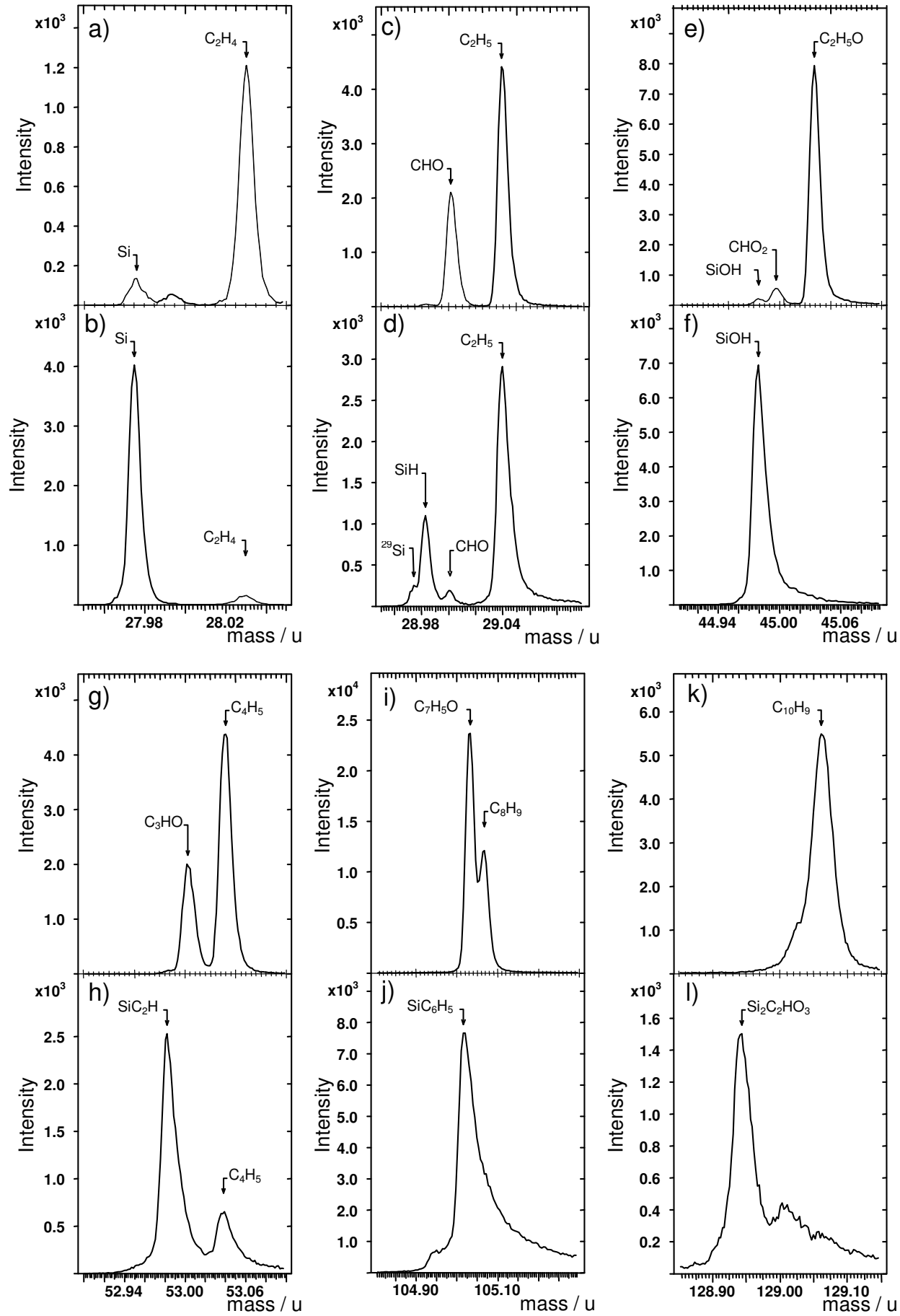

Figure 11. Comparison of positive ToF-SIMS spectra of a nanocomposite micro-CT fracture surface generated in UHV, a), c), e), g), i), k) and phenyl modified ormosil nanoparticles b), d), f), h), j), l) for nominal masses $28 \mathrm{u}, 29 \mathrm{u}, 45 \mathrm{u}, 53 \mathrm{u}, 105 \mathrm{u}$ and 129 u respectively. 


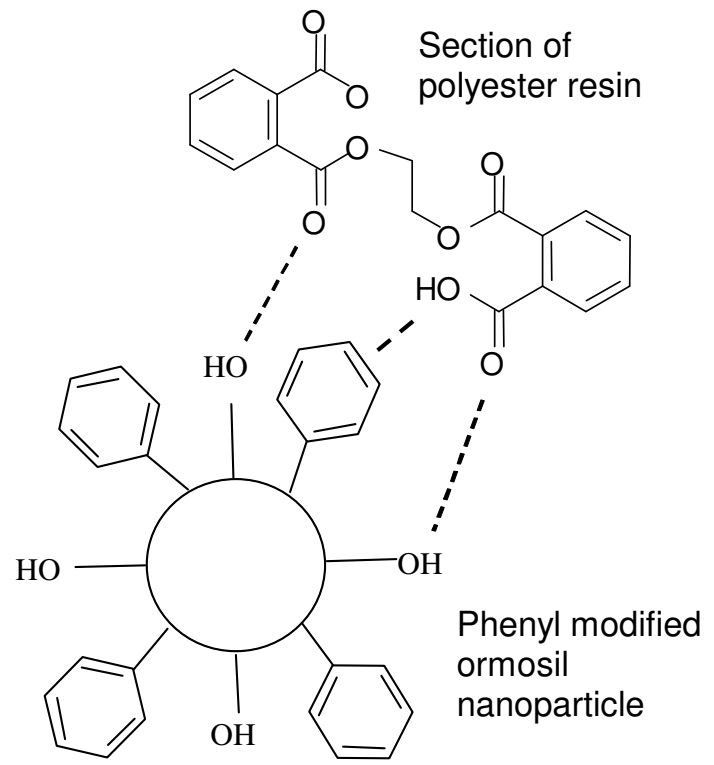

Figure 12. Schematic of the interaction between the phenyl modifed ormosil nanoparticle and a section of polyester resin, after Jesson et al [19] 
Table 1. Sample details

\begin{tabular}{|c|c|c|}
\hline Sample code & Material & Test conditions \\
\hline PRA & Plain resin & Air \\
\hline PRV & Plain resin & UHV \\
\hline NRA & Nanomodified resin & Air \\
\hline NRV & Nanomodified resin & UHV \\
\hline
\end{tabular}

Table 2. Polyester characteristic peaks; G (glycol), MA (maleic anhydride), TP (terephthalate)

\begin{tabular}{|c|c|c|c|}
\hline Nominal mass (u) & Fragment ion & Structure & Origin \\
\hline 39 & $\mathrm{C}_{3} \mathrm{H}_{3}^{+}$ & & $\mathrm{TP}$ \\
\hline 41 & $\mathrm{C}_{3} \mathrm{H}_{5}^{+}$ & $\mathrm{H}_{2} \mathrm{C}=\mathrm{CH}-\mathrm{CH}_{2}^{+}$ & G \\
\hline 45 & $\mathrm{C}_{2} \mathrm{H}_{5} \mathrm{O}^{+}$ & $-\mathrm{CH}_{2}-\mathrm{OH}$ & G \\
\hline 50 & $\mathrm{C}_{4} \mathrm{H}_{2}^{+}$ & & $\mathrm{TP}$ \\
\hline 51 & $\mathrm{C}_{4} \mathrm{H}_{3}{ }^{+}$ & & $\mathrm{TP}$ \\
\hline 65 & $\mathrm{C}_{5} \mathrm{H}_{5}^{+}$ & & $\mathrm{TP}$ \\
\hline 77 & $\mathrm{C}_{6} \mathrm{H}_{5}^{+}$ & & $\mathrm{TP}$ \\
\hline 91 & $\mathrm{C}_{7} \mathrm{H}_{7}^{+}$ & & MA \\
\hline 103 & $\mathrm{C}_{8} \mathrm{H}_{7}^{+}$ & & $\mathrm{TP}$ \\
\hline 105 & $\mathrm{C}_{7} \mathrm{H}_{5} \mathrm{O}^{+}$ & & $\mathrm{TP}$ \\
\hline 115 & $\mathrm{C}_{9} \mathrm{H}_{7}^{+}$ & & MA \\
\hline 128 & $\mathrm{C}_{10} \mathrm{H}_{8}^{+}$ & & MA \\
\hline 149 & $\mathrm{C}_{8} \mathrm{H}_{5} \mathrm{O}_{3}{ }^{+}$ & & $\mathrm{TP}$ \\
\hline 165 & $\mathrm{C}_{13} \mathrm{H}_{9}^{+}$ & & MA \\
\hline 178 & $\mathrm{C}_{10} \mathrm{H}_{10} \mathrm{O}_{3}^{+}$ & & $\mathrm{TP}$ \\
\hline 193 & $\mathrm{C}_{10} \mathrm{H}_{9} \mathrm{O}_{4}^{+}$ & & $\mathrm{TP}$ \\
\hline
\end{tabular}


Table 3. Phenyl ormosil characteristic peaks

\begin{tabular}{|c|c|c|}
\hline Nominal mass (u) & Fragment ion & Structure \\
\hline 28 & $\mathrm{Si}^{+}$ & $\mathrm{Si}^{+}$ \\
\hline 29 & $\mathrm{SiH}^{+}$ & ${ }^{+} \mathrm{SiH}$ \\
\hline 45 & $\mathrm{SiOH}^{+}$ & ${ }^{+} \mathrm{Si}-\mathrm{OH}$ \\
\hline 53 & $\mathrm{SiC}_{2} \mathrm{H}$ & ${ }^{+} \mathrm{C}$ \\
\hline 105 & $\mathrm{SiC}_{6} \mathrm{H}_{5}$ & ${ }^{+} \mathrm{Si}-\mathrm{C}-\mathrm{H}$ \\
\hline 129 & $\mathrm{Si}_{2} \mathrm{C}_{2} \mathrm{HO}_{3}$ & $\mathrm{HO}-\mathrm{Si} \equiv \mathrm{C}-\mathrm{O}-\mathrm{C} \equiv \mathrm{Si}-\mathrm{O}^{+}$ \\
\hline
\end{tabular}

21. 